\title{
AN ALTERNATIVE FORMULAE FOR THE NET PRESENT VALUE (NPV)
}

\author{
Hamed Habibi and Reza Habibi \\ PhD, Faculty of Science and Engineering, Curtin University, Perth, Australia \\ hamed.habibi@postgrad.curtin.edu.au \\ Iran Banking Institute, Central Bank of Iran, Tehran, Iran \\ r_habibi@ibi.ac.ir
}

\begin{abstract}
An alternative formulae is derived for NPV. First, the equivalence of NPV formulae and the value of a special portfolio is shown and weights of special portfolio is derived by minimizing the variance of portfolio.
\end{abstract}

KEYWORDS: Cash flow; Lagrange multiplier; NPV; NPVaR; Portfolio management

2010 AMS Mathematics Subject Classification: 62G20, 62F20

1. INTRODUCTION. The concept of NPV plays important role in finance. It is used to calculate the present value of financial projects and consequently to compare them. There are other concepts related to NNPV which may be used to compare projects. For example, Ye and Tiong (2000) introduced the concept of Net present value at risk (NPVaR) for financed infrastructure projects. For given discrete time cash flow stream $\left(i, F_{i}\right), i=1,2, \ldots$, the NPV is

$$
N P V=\sum_{i=1}^{\infty} \frac{F_{i}}{(1+r)^{i}}=Z\left(F_{i}\right)
$$

where $r$ is given interest rate and $Z$ is the $z$-transform function, see Park and Sharp-bette (1990). Let $\mathrm{E}\left(\mathrm{F}_{\mathrm{i}}\right)=\mu_{i}^{*}$, $\operatorname{var}\left(F_{i}\right)=\sigma_{i}^{* 2}$ and $\operatorname{cor}\left(F_{i}, F_{j}\right)=\rho_{i j}$. Assuming $F_{i}$ are independent variables, then

$$
E(N P V)=\sum_{i=1}^{\infty} \frac{\mu_{i}}{(1+r)^{i}} \text { and } \operatorname{var}(N P V)=\sum_{i=1}^{\infty} \frac{\sigma_{i}^{* 2}}{(1+r)^{2 i}} .
$$

The NPVaR is an aggregate risk measure for evaluating risk of projects. However, variation of NPV w.r.t its parameters is also regarded as individual risk measures like Greek letters in option pricing.

Proposition 1. The partial derivative of NPV w.r.t $\mathrm{r}$ is $\frac{\partial \mathrm{NPV}}{\partial \mathrm{r}}=\frac{-1}{(1+\mathrm{r})} \mathrm{Z}\left(\mathrm{iF}_{\mathrm{i}}\right)$, and notice that

$$
\frac{\partial E(N P V)}{\partial r}=\sum_{i=1}^{\infty} \frac{i \mu_{i}^{*}}{(1+r)^{i+1}} \text { and } \frac{\operatorname{var}(N P V)}{\partial r}=2 \sum_{i=1}^{\infty} \frac{i \sigma_{i}^{* 2}}{(1+r)^{2 i+1}} .
$$

There are many exact and approximate methods to derive the statistical characteristics of NPV. The following Remark mentions some of them.

Remark 1. The methods are

(i) Assuming normal distribution for NPV, then the sensitivity of NPVaR w.r.t the interest rate $r$ is computed.

(ii) If $\sigma_{i}^{* 2}$ constitute a time series structure (for example, $F_{i}$ be a return flow of a specified stock) then updating process like GARCH time series, Riskmetrics of Morgan or adaptive filters like Kalman filters may be applied to update $\sigma_{i}^{* 2}$.

(iii) Assuming distributions of $F_{i}$ 's are given, then the Monte Carlo simulation involved variance reduction may be used in Modelrisk software to calculate the NPVaR, as did Ye and Tiong (2000).

(iv) Beside this, the re-sampling method like bootstrapping and jackknife are applicable in this field.

(v) Assuming $r$ be a positive number and

$$
S_{n}^{2}=\sum_{k=1}^{n} \frac{\sigma_{i}^{* 2}}{(1+r)^{2 k}}
$$

then $\max _{1 \leq k \leq n} \frac{\sigma_{i}^{* 2}}{s_{n}^{2}}$ tends to zero as $n \rightarrow \infty$, then the Lindeberg-Feller central limit theorem condition (see Billingsley, 1986) is satisfied and the following variable converges to normal law in distribution, 
(vi) The beta approximation can be used to compute $E(N P V)$ and $\operatorname{var}(N P V)$.

(vii) An alternative approach to compute the NPVaR is to use the Chebyshev's inequality, although it uses the first two moments of NPV. However, Park and Sharp-bette (1990) suggested the Gauss-Camp-Meidall inequality which uses the first four statistical moment.

(ix) When $F_{i}$ 's are correlated then copula function may be applied to consider the correlation in $F_{i}$ 's. Hillier's method is an alternative method for correlated cash flows.

The following proposition gives an equivalent formulae for NPV. The proof is simple and is omitted.

Proposition 2. An equivalent formulae for NPV is

$$
N P V=\sum_{i=1}^{\infty} \frac{\sum_{j=1}^{i}\left(F_{j}-F_{j-1}\right)}{(1+r)^{i}}=\sum_{i=1}^{\infty} \frac{F_{i}-F_{i-1}}{r(1+r)^{i-1}} .
$$

Suppose that $F_{j}-F_{j-1}$ be a sequence of independent variables with mean $\mu_{i}$ variance $\sigma_{j}^{2}$. Therefore,

$$
E(N P V)=\sum_{i=1}^{\infty} \frac{\mu_{i}}{r(1+r)^{i-1}}, \operatorname{var}(N P V)=\sum_{j=1}^{\infty} \frac{\sigma_{j}^{2}}{r^{2}(1+r)^{2 i-2}} .
$$

2. ALTERNATIVE FORMULAE. Suppose that the NPV is defined as

$$
N P V=\sum_{i=1}^{\infty} a_{i} F_{i},
$$

for some sequence of real numbers $a_{i}$. Conditions on $a_{i}$ are found during the paper. Assume $F_{0}=0$ and write the NPV as

$$
\begin{gathered}
N P V=\sum_{i=1}^{\infty} a_{i} F_{i}=\sum_{i=1}^{\infty} \sum_{j=1}^{i} a_{i}\left(F_{j}-F_{j-1}\right) \\
=\sum_{j=1}^{\infty} \sum_{i=j}^{\infty} a_{i}\left(F_{j}-F_{j-1}\right)=\sum_{j=1}^{\infty} b_{j}\left(F_{j}-F_{j-1}\right),
\end{gathered}
$$

Where $b_{j}=\sum_{i=j}^{\infty} a_{i}$. Suppose that there is an portfolio with infinite assets at which each asset has $F_{j}$ value at time $j=1,2, \ldots$ and it has weight $b_{j}$. Suppose that $\sum_{j=1}^{\infty} b_{j}=1$.

Suppose that $F_{j}-F_{j-1}$ be a sequence of independent variables with variance $\sigma_{j}^{2}$. Therefore,

$$
\operatorname{var}(N P V)=\sum_{j=1}^{\infty} b_{j}^{2} \sigma_{j}^{2} .
$$

It is interested to minimize the $\operatorname{var}(N P V)$ w.r.t condition $\sum_{j=1}^{\infty} b_{j}=1$. The Lagrange multiplier function is

$$
L=\sum_{j=1}^{\infty} b_{j}^{2} \sigma_{j}^{2}-\varphi\left(\sum_{j=1}^{\infty} b_{j}-1\right) .
$$

The derivative of $L$ w.r.t $b_{j}$ is $2 b_{j} \sigma_{j}^{2}-\varphi \rho=0$, then

$$
b_{j}=0.5 \varphi \sigma_{j}^{-2} .
$$

Thus, $\varphi=\frac{2}{\sum_{j=1}^{\infty} \sigma_{j}^{-2}}$. It is seen that

$$
b_{j}=\frac{\sigma_{j}^{-2}}{\sum_{j=1}^{\infty} \sigma_{j}^{-2}} .
$$

Since $b_{j}-b_{j+1}=a_{j}$, then

$$
a_{j}=\frac{\sigma_{j}^{-2}-\sigma_{j+1}^{-2}}{\sum_{j=1}^{\infty} \sigma_{j}^{-2}} .
$$

The following proposition summaries the above discussion. 
Proposition 3. Assuming $F_{j}-F_{j-1}$ are independent random variables where $\sigma_{j}^{2}=\operatorname{var}\left(F_{j}-F_{j-1}\right)$, where

$$
\sum_{j=1}^{\infty} \sigma_{j}^{-2}<\infty
$$

Let $a_{j}=\frac{\sigma_{j}^{-2}-\sigma_{j+1}^{-2}}{\sum_{j=1}^{\infty} \sigma_{j}^{-2}}$. An alternative formulae for NPV is $N P V=\sum_{i=1}^{\infty} a_{i} F_{i}$.

Remark 2. The independency assumption happens rarely, in practice. For example, suppose that the correlation of $F_{i}-F_{i-1}$ and $F_{j}-F_{j-1}(i \neq j)$ be $\rho$. Also, suppose that $\sum_{i=1}^{\infty} b_{i} \sigma_{i}=g$. Let

$$
\lambda=\left(\sum_{j=1}^{\infty}{\sigma_{j}^{-2}}^{-2}\right)\left((2-\rho)+\rho g \sum_{j=1}^{\infty}{\sigma_{j}^{-1}}^{-1} .\right.
$$

Then, $b_{i}=\frac{\lambda-\rho g \sigma_{i}}{(2-\rho) \sigma_{i}^{-2}}$, and $b_{i}-b_{i+1}=a_{i}$.

Another approach is to minimize the variance of above portfolio while its mean is kept fixed in a specified level such as $c$. This NPV is called to mean-variance NPV (MVNPV). The following proposition summarizes the results:

Proposition 4. Let $F_{j}-F_{j-1}$ be independent variables with $\sigma_{j}^{2}=\operatorname{var}\left(F_{j}-F_{j-1}\right)$, and $\sum_{j=1}^{\infty} \mu_{j} \sigma_{j}^{-2}<\infty$. The MVNPV is given by $M V N P V=\sum_{i=1}^{\infty} a_{i}^{*} F_{i}$ where

$$
\mathrm{a}_{\mathrm{i}}^{*}=\frac{\mu_{j} \sigma_{j}^{-2}-\mu_{j+1} \sigma_{j+1}^{-2}}{\sum_{j=1}^{\infty} \mu_{j} \sigma_{j}^{-2}} .
$$

3. CONCLUDING REMARKS. It was shown that the NPV may be represented as the gain (or loss) of a portfolio and this note is used to derive another versions for NPV.

\section{REFERENCES}

[1] Billingsley, P. 1986. Probability and Measure . Wiley.

[2] Park, C. S. and Sharp-bette, G. P. 1990. Advanced Engineering Economics. Wiley.

[3] Ye, S. and Tiong, R. L. K. 2000. NPV-at-risk method in infrastructure project investment evaluation. Journal of Construction Engineering and Management 7, 227-233. 